\title{
Granulicatella elegans bacteremia \& meningitis in a child without neurosurgical interventions
}

\author{
Dr Sowmya G Shivappa ${ }^{1}$, Dr Madhuri Kulkarni ${ }^{2}$, Dr Manjula S. R ${ }^{3}$, \\ Dr Bewin Oral ${ }^{4}$ \\ ${ }^{1}$ Assistant Professor, Department of Microbiology, JSS Medical College, JSS University, Mysore, India \\ ${ }^{2}$ Professor \& Head, Department of Microbiology, JSS Medical College, JSS University, Mysore, India \\ ${ }^{3,4}$ Post graduate, Department of Microbiology, JSS Medical College, JSS University, Mysore, India
}

\begin{abstract}
Granulicatella elegans is a nutritionally variant streptococci generally associated with infective endocarditis. Meningitis due to G.elegans is very rare and it has always occurred in cases following neurosurgical procedures. We report the case of acute pyogenic meningitis with bacteremia with parenteral diarrhea in a ten month old immunocompetent child without any preceding neurosurgical intervention or dental manipulations. This case emphasizes the need of high degree clinical suspicion, use of appropriate culture media for isolation and identification of G.elegans and rapid initiation of adequate antibiotic therapy.
\end{abstract}

Keywords: Bacteremia, Granulicatella elegans, Meningitis, Nutritionally variant Streptococci

\section{Introduction}

Nutritionally Variant Streptococci (NVS) are Gram Positive Cocci first identified in 1961 from a patient with Infective endocarditis by Frenkel and Hirsch [1]. NVS are normal flora of the upper respiratory, urogenital and gastrointestinal tracts of humans [2]. They cause sepsis and bacteremia and are isolated as causes of various infections like infective endocarditis, otitis media, keratoconjunctivitis, pancreatic abscess, total knee arthroplasty infection, osteomyelitis, intramammary foreign body infection, postpartum sepsis and central nervous system infections.

The two important genera of NVS are Abiotrophia (A. defectiva) and Granulicatella (G. adiacens, G. balenopterae and G. elegans) [3]. Infections by Granulicatella most commonly occur in patients with underlying cardiac disease, febrile neutropenia or chemotherapy associated mucositis and neutropenia. Higher rates of orointestinal colonization by Granulicatella and subsequent mucositis may predispose for bacteremia in such patients [4].

The isolation of NVS is difficult, since they do not grow on ordinary culture media and requires the use of media supplemented with pyridoxal and L-cysteine or grow faintly on chocolate agar [2]. Fastidious nature of the growth and high prevalence of beta lactum, and macrolide resistance pose challenge to treat invasive infections caused by these NVS [5]. In this paper, we describe the case of a ten months old child with acute pyogenic meningitis with septicemia with parenteral diarrhea caused by Granulicatella elegans.

\section{Case report}

A ten months old male child was brought to Paediatric OPD with complaints of fever, vomiting and loose stools of 3 days duration. Fever was high degree $\left(104^{\circ} \mathrm{c}\right)$ intermittent type associated with chills. The child passed 25-30 loose stools/day, watery, foul smelling and non blood tinged, associated with nausea and vomiting. The child was born to a non consanguinous couple by caesarean section at 40 weeks because of the presence of cord around the neck with a birth weight of $3.9 \mathrm{kgs}$. There was no abnormality in the prenatal, natal or postnatal period.

On examination, the child was conscious and irritable. Liver was palpable $3 \mathrm{~cm}$ below the right costal margin. Slight bulge of the anterior fontanelle was observed and fundoscopy revealed signs of early papilloedema. Other signs of meningeal irritation were not seen and cardiovascular and respiratory system was normal.

Laboratory investigations revealed normal haematological parameters, liver \& renal function tests and normal serum electrolytes. Urine microscopy and culture examination was normal. CSF examination showed 80 cells/cumm, predominantly neutrophils and occasional lymphocytes. CSF culture did not yield any bacterial growth. CSF glucose was $25 \mathrm{mg} / \mathrm{dl}$ and protein was $120 \mathrm{mg} / \mathrm{dl}$. Stool microscopic examination showed no inflammatory cells or red blood cells or parasitic forms and culture did not yield the growth of any pathogenic bacteria.

Two sets of blood cultures were collected 24 hours apart before the administration of antibiotics and processed by BacT/ALERT Microbial Detection system (bioMerieux, Inc). Both the samples flagged positive within 24 hours of incubation. Gram staining of the direct smear from blood culture showed pleomorphic Gram 
positive cocci in chains, pairs and coccobacillary forms. Subculture was done on $5 \%$ sheep blood agar and chocolate agar and plates were incubated for 48 hours in $10 \% \mathrm{CO}_{2}$. Weak growth was observed in the first 24 hours and the growth became more obvious after 48hours [Fig 1]. Tiny $(<0.2 \mathrm{~mm})$ non haemolytic grayish white colonies were observed which were catalase and oxidase negative and resistant to optochin. Gram stain smear from blood agar showed Gram Positive coccoid forms in pairs and short chains [Fig 2]. Vitek 2 Compact system (Biomerieux) identified the isolate as Granulicatella elegans with $99 \%$ probability value. Pyrrolidonyl arylamidase, leucine arylamidase, alanine arylamidase, tyrosine arylamidase, L-proline arylamidase and arginine dihydrolase tests were positive and $\alpha \& \beta$ galactosidase, $\beta$ glucuronidase, esculin hydrolysis and urease tests were negative [6]. Antimicrobial susceptibility was performed using Kirby Bauer disc diffusion method according to standard guidelines [7]. The isolate was susceptible to ampicillin, erythromycin, cotrimoxazole, ciprofloxacin, gentamicin, ceftriaxone, ceftazidime, piperacillin tazobactum, linezolid, vancomycin and teicoplanin.

The child was successfully treated with intravenous amikacin $75 \mathrm{mg}$ twice daily and intravenous ceftriaxone $500 \mathrm{mg}$ twice daily for ten days supported with paracetamol, domperidone, probiotics, oral rehydration salts and intravenous fluids. The child became afebrile from $4^{\text {th }}$ day after starting antibiotics. Vomiting and loose stools also subsided subsequently and the child was discharged after complete clinical recovery.

\section{Discussion}

Granulicatella elegans is a fastidious Gram Positive coccus belonging to genus Granulicatella, which is a Nutritionally Variant Streptococcus. Because of their fastidious nutritional requirements, they have been variously termed as Nutritionally deficient streptococci, Satelliting streptococci, Vitamin $\mathrm{B}_{6}$ dependent streptococci, Pyridoxal dependent streptococci or Cell wall deficient forms (L forms) [3].

The isolation and identification of the species is difficult as the bacteria exhibits variability and pleomorphism on Gram staining, ranging from cocci arranged in short chains in nutrition rich media to swollen bacillary forms in nutrient depleted media $[2,6]$. This variability poses challenges to the identification and taxonomic classification of the organism. The possibility of nutritionally variant Streptococci should be considered when Gram positive cocci or bacilli are seen on Gram staining and the organism grows poorly on non supplemented media. Also these isolates can naturally exist in cell wall deficient state (L forms) and may be difficult to isolate from clinical specimens such as blood cultures [1].

Studies have shown that addition of $0.001 \%$ of pyridoxal $\mathrm{HCl}$ facilitates the growth of NVS. Pyridoxal is required for coenzymatic transformation of L-alanine to D-alanine, which is necessary for peptidoglycan production [2]. This causes most isolates to convert to streptococcus like cellular arrangement and Gram positivity [6]. Chocolate agar contains little pyridoxal from red cell hemolysis, on which weak growth can be observed. In our case the BacT/ALERT media which is enriched with $0.001 \%$ pyridoxal, and $0.025 \% \mathrm{~L}$-cysteine helped in the isolation of Granulicatella elegans species.

The rate of oral colonization by Granulicatella is more than that of Abiotrophia spp. About $10 \%$ of the adult dental plaque is colonized with $\mathrm{G}$ elegans [8]. The tooth-tissue interface is a common portal for entry of bacteria into body. Bacteremia can occur following dental manipulations [5], tonsillectomy, bronchoscopy, and transient bacteremia can occur following tooth brushing, poor dental hygiene [9] or even by chewing gum [10]. Hematogenous translocation of maternal oral flora into the amniotic environment as the likely source of neonatal bacteremia has also been reported [11]. Our patient did not have any history of dental treatment or invasive procedures. Poor oral hygiene and teething might have resulted in bacteremia in our case.

Bacteremia is one of the most common predisposing factors for meningitis [12]. In our case, G. elegans bacteremia has caused meningitis, but CSF microscopic examination and culture were negative, as CSF was collected after two days of antibiotic administration. But reduced CSF glucose level and slightly elevated protein level were suggestive of acute pyogenic meningitis. Granulicatella infections following neurosurgery have been reported to cause meningitis and epidural abscess [13], but meningitis in a patient without any neurosurgical intervention has not been described. There were no predisposing factors for meningitis in our case. Brain abscess without neurosurgery in an immunologically intact adult have also been described [14].

Meningitis in children is usually preceded by several days of fever accompanied by upper respiratory tract or gastrointestinal symptoms, followed by nonspecific signs of CNS infection such as increasing lethargy and irritability [12]. Our case also presented with predominant symptom of watery diarrhea, fever and irritability. Hence gastrointestinal complaints have to be thoroughly evaluated as a sign of severe systemic diseases like meningitis.

Most common clinical condition caused by G. elegans is Infective endocarditis $[6,8,15]$ followed by bacteremia [11]. They have also been isolated from other infections like otitis media [1], keratoconjunctivitis [16], pancreatic abscess [17], osteomyelitis, total knee arthroplasty infection [18], breast implant infection [19] and postpartum sepsis [20]. 
High prevalence of beta lactam $(50 \%)$ and macrolide resistance $(54 \%)$ pose challenge in treating invasive infections [5]. Various susceptibility patterns to aminoglycosides have also been reported [8]. Little is known about the genetic basis of their resistance mechanisms. Our isolate was sensitive to beta lactams, macrolides, fluroquinolones, aminoglycosides, cephalosporins, linezolid, vancomycin and teicoplanin. Long term combination therapy with beta lactam and an aminoglycoside is usually recommended. Treatment failures with monotherapy are known [9]. In our case, the child was treated with a combination of Amikacin and ceftriaxone with complete clinical recovery.

\section{Conclusion}

Granulicatella is an important agent causing bacteremia, infective endocarditis \& meningitis with increased morbidity and mortality. Its fastidious nature of growth has led to difficulties in isolation and identification in clinical microbiology laboratories. Hence appropriate culture techniques and high degree of suspicion in clinical samples is necessary for isolation and identification. Also significant resistance to beta lactams and macrolides emphasize the need for antimicrobial susceptibility testing and early administration of long term combination therapy.

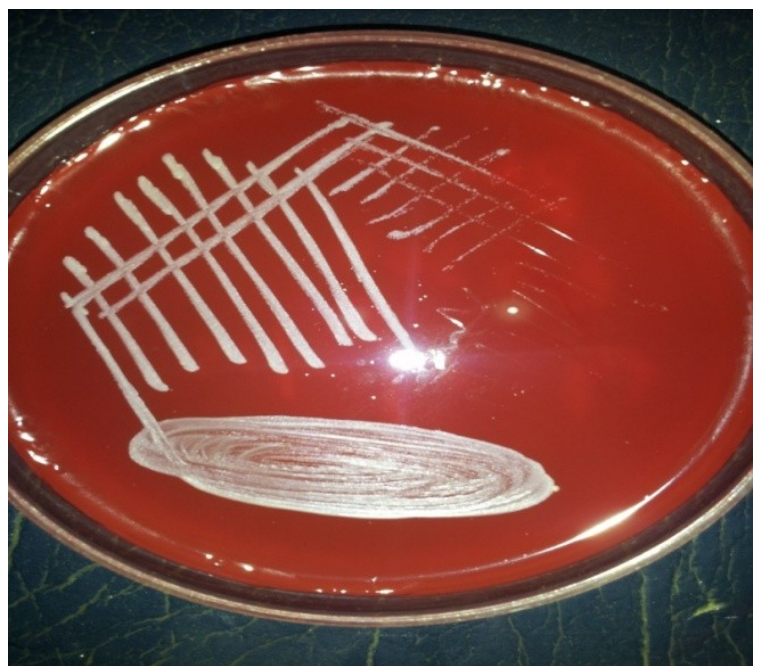

Fig 1: Colony morphology of G. elegans on Blood agar

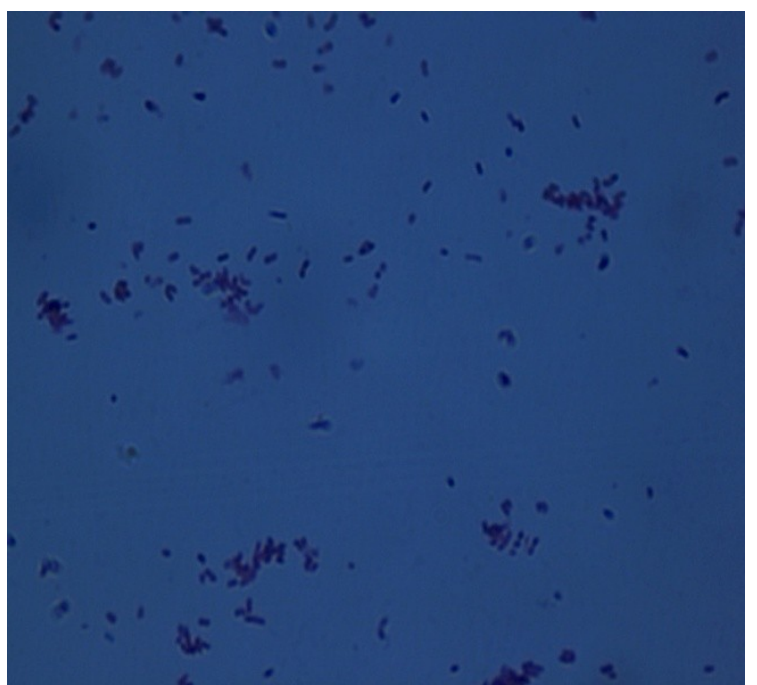

Fig 2: Gram stain morphology of G. elegans

\section{References}

[1] Frenkel A, Hirsch W. Spontaneous development of L forms of streptococci requiring secretions of other bacteria or sulphydryl compounds for normal growth. Nature. 1961; 191:728-730.

[2] Rouff KL. Nurtritionally variant streptococci. Clin Microbiol Rev. 1991;4:184-190.

[3] Collins MD, Lawson PA. The genus Abiotrophia(Kawamura et al.) is not monophyletic: proposal of Granulicatella gen. nov., Granulicatella adiacens comb. Nov., Granulicatella elegans comb. Nov. and Granulicatella balaenopterae comb. Nov. Int J Syst Evol Microbiol 2000;50:365-369. 
[4] Senn L, Entenza JM, Greub G et al. Bloodstream and endovascular infections due3 to Abiotrophia defective and Granulicatella species. BMC Infect Dis. 2006;20:6:9.

[5] Liao CH, Teng LJ, Hsueh PR, Chen YC, Huang LM, Chang SC et al., Nutritionally variant Streptococcal infections at a University Hospital in Taiwan: Disease emergence and high prevalence of $\beta$ Lactam and Macrolide resistance. Clinical Infectious Diseases. 2004;38:452-455.

[6] Christensen JJ, Facklam RR. Granulicatella and Abiotrophia species from human clinical specimens. Journal of Clinical Microbiology. 2001; 39:3520-3523.

[7] Committee for Clinical Laboratory Standards Institute. 2005. Performance standards for antimicrobial disk susceptibility testing, fifteenth informational supplement. Approved standard M2-A8. National Committee for Clinical Laboratory Standards, Wayne, Pa.

[8] Nemoto YO, Kishi K, Satho M, Tajika S, Sasaki M, Namioka A et al., Infective Endocarditis caused by Granulicatella elegans Originating in the Oral cavity. Journal of Clinical Microbiology. 2005;43(3):1405-1407.

[9] Vandana KE, Mukhopadhyay C, Rau NR, Ajith V, Rajath P. Native valve endocarditis and femoral embolism due to Granulicatella adiacens: a rare case report. Brazilian Journal of Infectious Diseases. 2010;14(6):634-636.

[10] Cobe HM. Transitory bacteremia. Journal of Oral Surgery. 1954;7:609-615.

[11] Quartermain L, Tailor H, Njenga S, Bhattacharjee P, Rao GG. Neonatal Granulicatella elegans Bacteremia, London, UK. Emerging Infectious Diseases. 2013;19(7):1165-1166.

[12] Charles G, Prober, Dyner L. Acute Bacterial Meningitis beyond the neonatal period. In: Kliegman, Stanton, St. Geme, Schor, Behrman, editors. Nelson Textbook of Pediatrics. $19^{\text {th }}$ ed. Philadelphia: Elsevier; 2012: 2087-2095

[13] Cerco E, Christie JD, Nachamkin I et al., Central Nervous System infections due to Abiotrophia and Granulicatella species: An emerging challenge? Diagnostic Microbiology and Infectious disease. 2004;48:161-165.

[14] Levin, Yana D, Petronaci, Lynn C. Isolation of Abiotrophia/Granulicatella species from a brain abscess in an adult patient without prior history of neurosurgical instrumentation. Southern Medical Journal. 2010;103(4):386-387.

[15] Casalta JP, Habib G, Scola B La, Drancourt M, Caus T and Raoult D. Molecular diagnosis of Granulicatella elegans on the Cardiac Valve of a Patient with Culture-negative Endocarditis. Journal of Clinical Microbiology. 2002;40(5):1845-1847.

[16] Barrios H, Bump CM. Conjunctivitis caused by a nutritionally variant streptococcus. Journal of Clinical Microbiology. 1986;23:379-380.

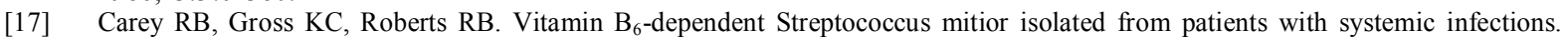
Journal of Infectious Diseases. 1975;131:722-726.

[18] Ince A, Tiemer B, Gille J, et al. Total knee arthroplasty infection due to Abiotrophia defective. J Med Microbiol. 2002;51:899-902.

[19] Del Pozo JL, Garcia-Qetglas E, Hernaez S, et al. Granulicatella adiacens breast implant associated infection. Diagnostic Microbiology and Infectious disease. 2008;61:58-60.

[20] McCarthy LR, Bottone EJ. Bacteremia and endocarditis caused by satelliting streptococci. Am J Clin Pathol. 1974;61:585-591. 